\title{
SPECTRAL FEATURES OF THE SYMBIOTIC VARIABLE STAR CH CYGNI IN 2005 - 2006
}

\author{
Kye Hwa Yoo ${ }^{1}$ and Tae Seog Yoon ${ }^{2}$ \\ ${ }^{1}$ Department of Science Education, Ewha Womans University, Seoul, 120-750, Korea \\ E-mail:khyoo@ewha.ac.kr \\ 2 Department of Astronomy and Atmospheric Sciences, Kyungpook National University, Daegu, 702-701, Korea \\ E-mail:yoonts@knu.ac.kr
}

(Received June 12, 2009; Accepted July 28, 2009)

\begin{abstract}
This article reports the spectral behavior of $\mathrm{CH}$ Cygni, using data obtained in October 2005 and June 2006. In these epochs, CH Cygni showed emission lines of H I, Fe II, [Fe II], [O III], [N II], [Ne III] and [S II]. Many of these lines were more enhanced since 2004. The underlying M-type spectrum was removed to get the intrinsic emission profile, and the resulting profiles were deconvoluted into several Gaussian components. Also, the radial velocities for all the lines that appeared in these spectra of $\mathrm{CH}$ Cygni were measured. The resultant lines were compared with each other and with those obtained in 2004; the findings are explained in terms of an accretion disk and jets.
\end{abstract}

Key words : Symbiotic variable star CH Cygni, enhancement of spectral lines, accretion disk, jets

\section{INTRODUCTION}

Before 1963, CH Cygni was classified as an M7 IIItype (Yamashita, 1967). And, the first eruption of CH Cygni was observed in 1963 (Faraggiana and Hack, 1969). At that time, the spectra of CH Cygni showed the blue continuum and emission lines of $\mathrm{H} \mathrm{I}$, He I and [Fe II] overlain on the M III-type spectrum. In 1963 2000, a symbiotic variable star CH Cygni had several outburst phases (Yamashita and Maehara, 1979; Mikolajewski et al., 1990; Eyres et al., 2002; Skopal et al., 2002). In the eruption and quiescence phases, $\mathrm{CH}$ Cygni was observed on X-ray, UV, visible, IR and radio wavelengths (Yoo, 2006, Paper I, references in there). While the broadest emission component of $\mathrm{H}$ I lines was caused by bipolar radio jets (Paper I), Contini et al. (2008) proposed the broadest emission component of $\mathrm{H}$ I lines was formed from downstream of a fast reverse shock.

Two models of $\mathrm{CH}$ Cygni have been proposed; one is the binary system with the orbital period of 5750day which consists of an M III and a white dwarf (Yamashita and Maehara, 1979), and the other is the triple system with orbital periods of 756-day and 5300-day which consists of an M III, a white dwarf and an unseen object (Hinkle et al., 1993).

In 2004, CH Cygni had $\mathrm{m} \sim 8.1$ in the $\mathrm{V}$ band, whereas the magnitudes of $\mathrm{CH}$ Cygni in the $\mathrm{V}$ band in October 2005 and June 2006 were $\mathrm{m} \sim 7.9$ and $\sim$ 8.2, respectively (AAVSO). In the period between October 2005 and June 2006 (called 'the present epochs' hereafter), the $\mathrm{TiO}$ bands and metal absorption lines

Corresponding Author: K.-H. Yoo of the M-type giant appeared to have nearly the same strength as those in 2004. However, weaknesses of the M-type lines were observed in the outburst phases.

In Paper I and Paper II (Yoo, 2007), detailed information on the profiles and radial velocities for the lines of CH Cygni observed in April 2004 and October 2004 were presented, respectively.

In this Paper, the variations in the spectral lines of the high-resolution spectra secured in October 2005 and June 2006 using the spectrograph of the Bohyunsan Optical Astronomy Observatory (BOAO) are reported. In the second section, the observations and data reduction procedures are described. In the third section, the behaviors of the spectral lines observed in the present epochs are detailed in comparison with the behaviors of those obtained in 2004. Subtraction of the spectrum of the underlying M-type giant is provided in the fourth section. Gaussian deconvolutions for $\mathrm{H} \alpha$, [O III], [Ne III], [N II], and [S II] and the radial velocity measurements are furnished in the fifth and sixth sections, respectively. In the last section, the line features are discussed in detail and a conclusion is arrived at.

\section{OBSERVATIONS AND DATA REDUC- TIONS}

Observations were carried out on October 13, 2005, and June 4, 2006, with a high resolution Echelle spectrograph, BOES (BOAO Echelle Spectrograph), mounted on an $1.8 \mathrm{~m}$ reflector at the BOAO. A $2048 \times 4096$ pixel CCD camera, with a size of $15 \times 15$ $\mu \mathrm{m}$ per pixel, was used. The spectral ranges used were from 3800 - $7000 \AA$. A cross disperser divides the spec- 
TABLE 1.

JoURNAL OF OBSERVATIONS OF CH CYGNI

\begin{tabular}{ccccccc}
\hline \hline $\begin{array}{c}\text { File } \\
\text { number }\end{array}$ & $\begin{array}{c}\text { JD } \\
2450000\end{array}$ & $\begin{array}{c}\text { Date } \\
\text { UT }\end{array}$ & $\begin{array}{c}\text { Phase } \\
\phi\end{array}$ & $\begin{array}{c}\text { Exposure } \\
\text { time }(\mathrm{sec})\end{array}$ & $\begin{array}{c}\text { Diameter of } \\
\text { fiber }(\mu \mathrm{m})\end{array}$ & $\begin{array}{c}\text { Weather } \\
\text { condition }\end{array}$ \\
\hline es16335 & 3657.013 & Oct. 13, 2005 & 0.52 & 1000 & 200 & poor \\
es22773 & 3892.096 & June 4, 2006 & 0.57 & 1280 & 300 & poor \\
\hline \multicolumn{7}{l}{ Phases are cited from the ephemeris of Hinkle et al.(1993). }
\end{tabular}
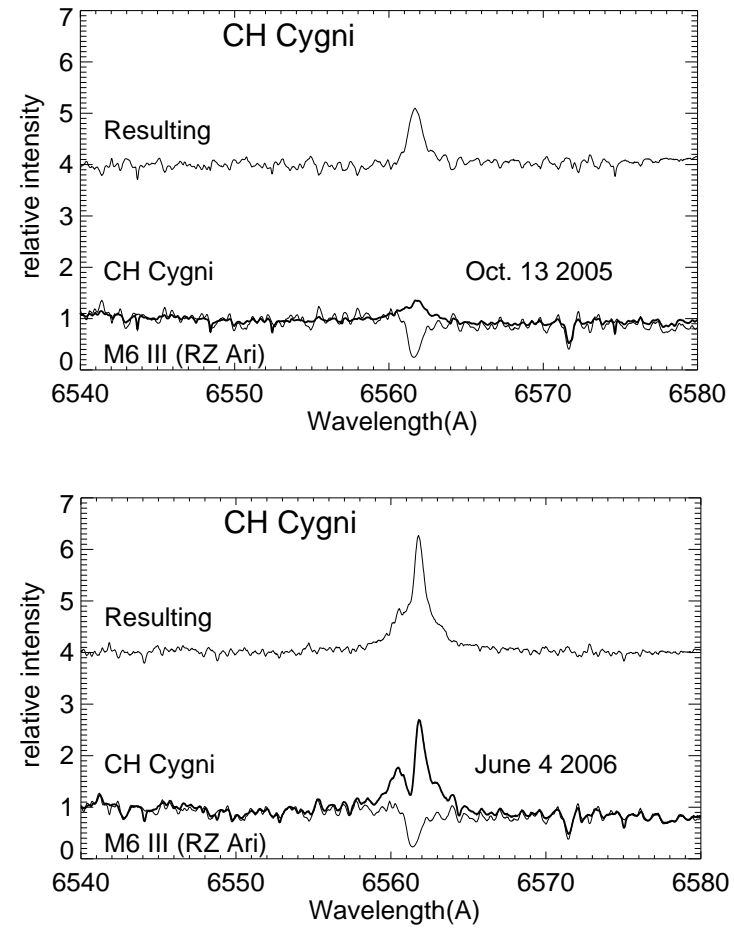

Fig. 1. - The spectra around $\mathrm{H} \alpha$ on October 13, 2005 (the upper panel), and on June 4, 2006(the lower panel) after the subtraction of the M-type spectrum.

tra into all 28 dispersion orders. An observation journal is outlined in Table 1.

The nominal spectral resolving powers, $\lambda / \Delta \lambda$, were 45000 for the $200 \mu \mathrm{m}$ fiber and 30000 for the $300 \mu \mathrm{m}$ fiber. The output dispersions covered a range from $1.56-2.14 \AA \mathrm{mm}^{-1}$, relied on the wavelength.

Data were reduced with the NOAO IRAF package involving bias, flat-field and spectra extraction processes. Both the pixel positions and the wavelengths were related to their 6th-order polynomials.
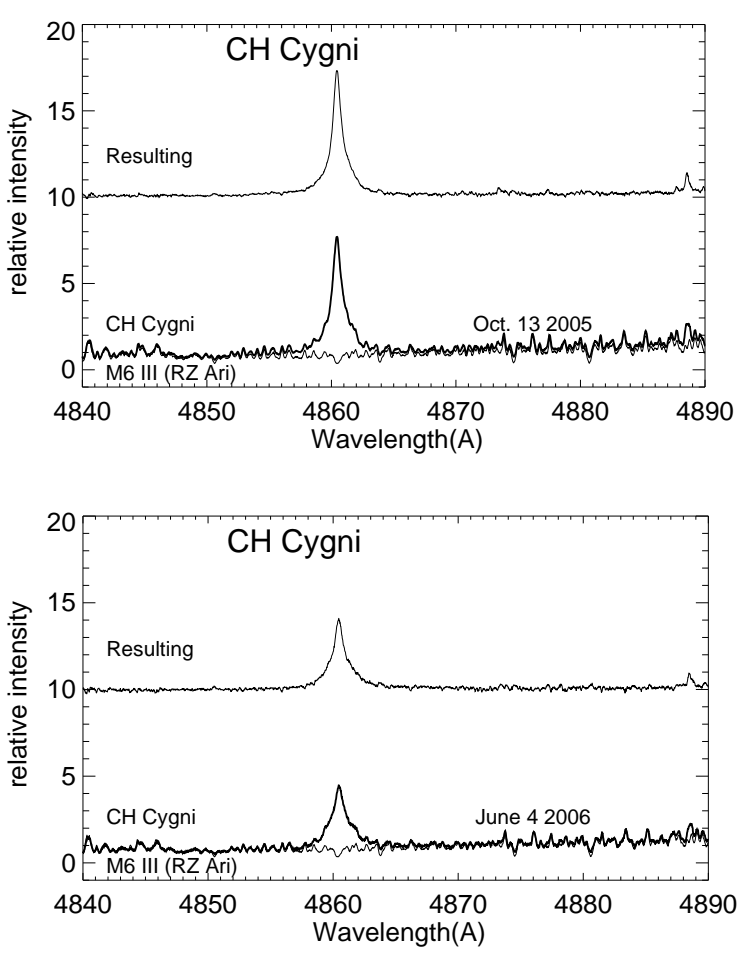

Fig. 2.- The spectra around $\mathrm{H} \beta$ on October 13, 2005 (the upper panel), and on June 4, 2006 (the lower panel) after the subtraction of the M-type spectrum.

\section{SPECTRAL VARIATIONS}

The lower portion of each panel in Figs. 1 - 5 is explained in this section. It is noticed here that all the spectra represented in Figs. 1 - 5 were made use of in the normalized intensity scale. The units of the abscissa in Figs. 1 - 5 are wavelengths, all values being corrected to the sun.

In the present epochs, the $\mathrm{H} \alpha$ line was changed from a very weak single-peaked emission profile (in October 2005) into a double-peaked line (in June 2006). $\mathrm{H} \beta$ showed a single-peaked emission profile during the present epochs, which corresponded with the redward emission component observed in April 2004, and the 

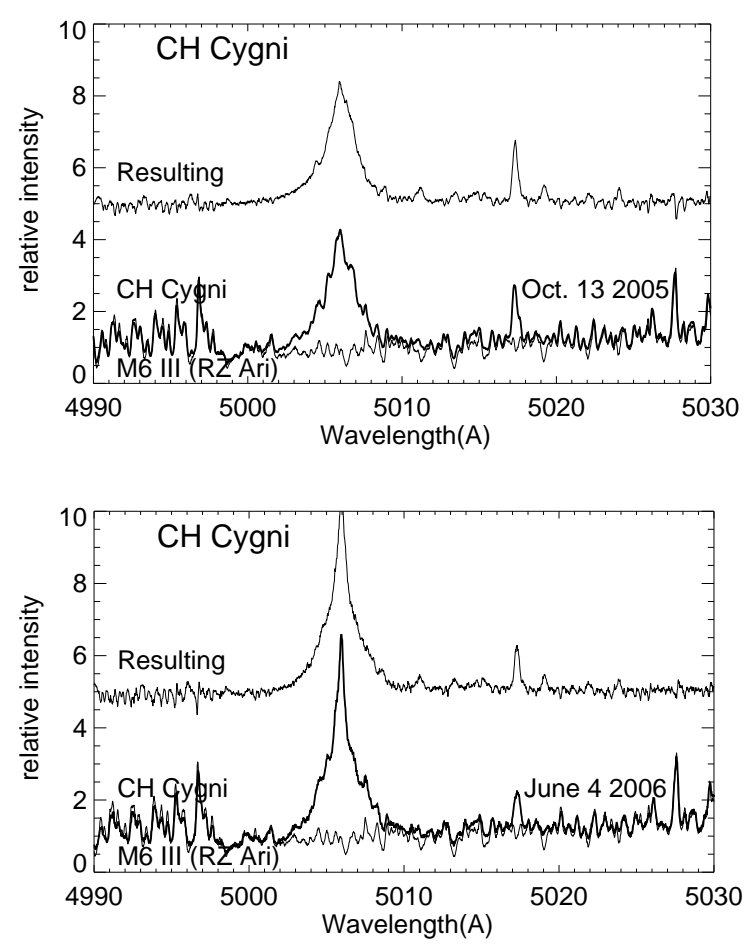

Fig. 3.- The spectra around [O III] $5007 \AA$ on October 13, 2005 (the upper panel), and on June 4, 2006 (the lower panel) after the subtraction of the M-type spectrum.

profile of $\mathrm{H} \beta$ in 2004 was stronger than $\mathrm{H} \alpha$ in October 2005. The $\mathrm{H} \epsilon$ line was very weak, although it might have blended with $\mathrm{Ca}$ II $\mathrm{H}$ line. $\mathrm{H} 8$ and $\mathrm{H} 9$ lines appeared to be very weak. The line profiles of $\mathrm{H} \alpha$ and $\mathrm{H} \beta$ in the present epochs are shown in the lower parts of each panel of Figs. 1 and 2 .

In early 2004, $\mathrm{H} \alpha$ and $\mathrm{H} \beta$ had double-peaked profiles, and in late 2004 , they were altered into singlepeaked profiles. On the contrary hand, in the 1977 outburst phase, they always showed double-peaked emission profiles, and around the end of the outburst phase, the blueward emission component became weak, with the absorption component around its line center becoming gradually weakened and finally disappearing (Yamashita and Maehara, 1979; Hack et al., 1986; Hack et al., 1988).

The main emission components of [O III] $4959 \AA$ and $5007 \AA$ were gradually more enhanced during the present epochs and turned more intense than those in 2004. However, [O III] $4363 \AA$ did not appear during the present phases.

Broad [Ne III] $3869 \AA$ appeared to present three components after 2004. [Ne III] and [O III] began to appear around the end of the outburst phases (Hack et al., 1988).

After subtracting the spectrum of the M-type giant, which is described in the next section, [N II] $6445 \AA$
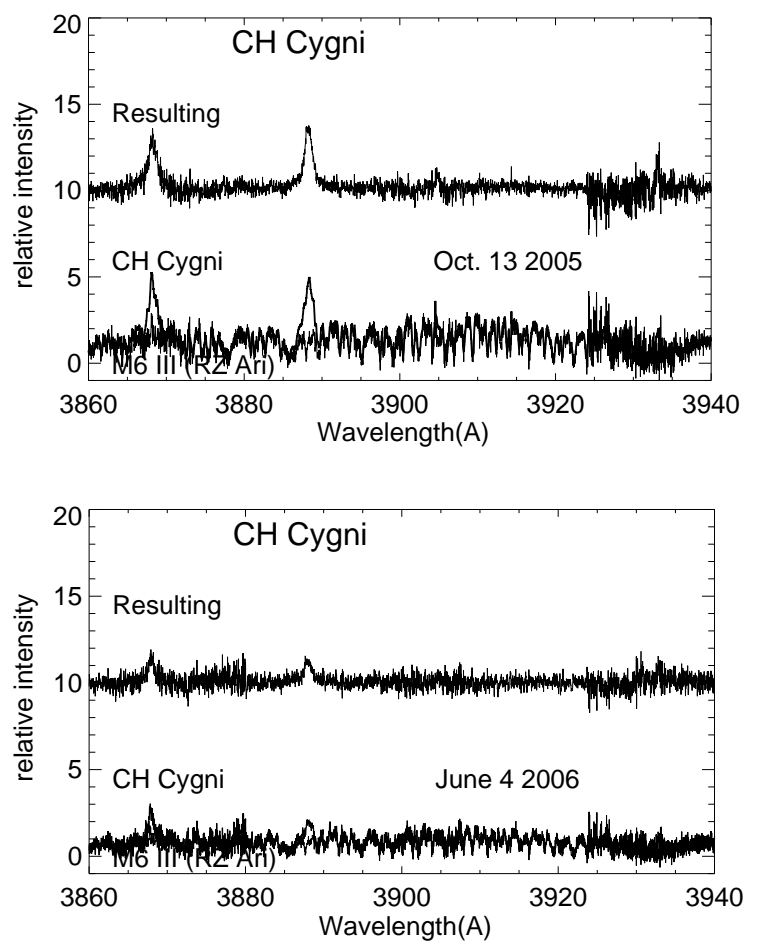

Fig. 4. - The spectra around [Ne III] $3869 \AA$ on October 13, 2005 (the upper panel), and on June 4, 2006 (the lower panel) after the subtraction of the M-type spectrum.

and $6584 \AA$ were clearly observed in 2004 , whereas they were not detected in 2005. In 2006, they had profiles that were more enhanced than in 2004.

[S II] $4069 \AA$ and $6584 \AA$ could be definitely confirmed in the spectra resulting from the subtraction of the spectrum from the M-type giant in October 2005 and June 2006. This indicates that in these epochs, the [S II] matter surrounding CH Cygni was either not expanding out or not flowing into the region of $[\mathrm{S} \mathrm{II}]$ formation. The line profiles of [O III] $5007 \AA$, [Ne III] $3869 \AA$, and [S II] $6584 \AA$ are presented in the lower panels of Figs. 3 - 5, respectively.

On June 4, 2006, the peak intensity of [O I] $6300 \AA$ was stronger by about 2 times than that on October 13 , 2005. In 2004, its intensity had remained unchanged for one year.

Ca II H and K lines had P Cygni profiles in October 2004 and in October 2005. However, in June 2006 these lines had a single emission. Na I D lines had two emission components after October 2004 when the spectrum of the underlying M-type giant was subtracted. Since 2004, their intensities continued to be increased. The absorption line components of $\mathrm{Ca}$ II $\mathrm{H}$ and $\mathrm{K}$ lines, and $\mathrm{Na} I \mathrm{D}$ lines were already described in Paper II.

Fe II $5018 \AA$ lines were present around $5018 \AA$ (Fig. $3)$. [Fe II] continued to remain intense from 2004 to 2006. However, in the present epochs, fewer Fe II lines 

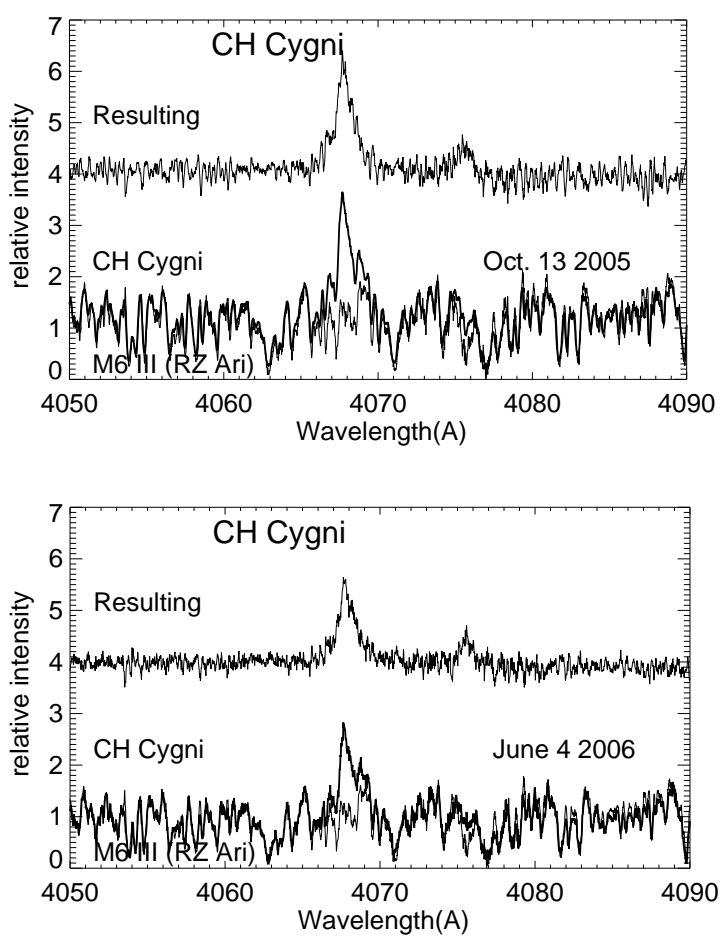

Fig. 5. - The spectra around [S II $] 4069 \AA$ on October 13, 2005 (the upper panel), and on June 4, 2006 (the lower panel) after the subtraction of the M-type spectrum.

were identified than [Fe II]. Moreover, the Fe II line profiles were asymmetric.

Many $\mathrm{TiO}$ bands were observed in the strong absorption spectra. Since 2004, the depth of the $(4,0)$ band head of the $\alpha$-system of $\mathrm{TiO}$ at $4424 \AA$ in these phases became gradually shallow, but it was still alive. The depth of the $\mathrm{TiO}$ band head at $4424 \AA$ in October 2004 was deeper by about 2 times than that in October 2005, and the depth in October 2005 was comparable to that in June 2006.

Since 2004, the depth of the Ca I $6573 \AA$ line of the M-type giant became gradually weak, whereas that of the Sr II $4215 \AA$ line remained nearly unchanged.

In 2004 - 2006, behaviors of spectral lines of $\mathrm{CH}$ Cygni is summarized as follows. $\mathrm{H} \alpha$ showed a singlepeaked emission line in October 2005, which subsequently changed into a double-peaked profile in June 2006 , with no absorption line observed at its linecenter. In April 2004, $\mathrm{H} \alpha$ and $\mathrm{H} \beta$ showed doublepeaked emission profiles with an absorption line around each of their line-centers. In the present epochs, nebular emission lines of [O III] and [Ne III] were produced; in addition, more Fe II and [Fe II] lines were observed than in 2004 Paper I; Paper II . Ti II and Cr II observed during the past outburst phases were absent in the present epochs. Moreover, in the present epochs, emission lines of He I, [O I] and [O III] were observed

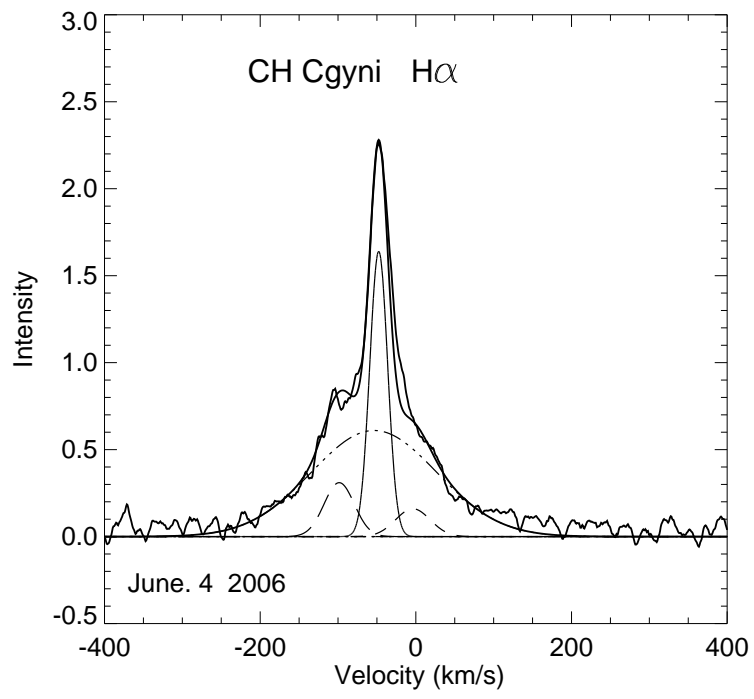

Fig. 6. - Gaussian deconvolution for the resultant $\mathrm{H} \alpha$ on June 4, 2006. The solid line denotes the observed line. The bold solid, the solid, the dashed, the dotted, and the dash dotted lines mean the result of fitting and each Gaussian component. Comparison of Gaussian profiles and a synthetic rectangle-like line profile would be noticed. The red line profile representing a theoretical model for the accretion disk is over-plotted.

and Na I D had double absorption cores.

\section{SUBTRACTION OF THE UNDERLY- ING M-TYPE SPECTRUM}

On October 13, 2005, and June 4, 2006, CH Cygni spectra were compounded with emission lines and the spectrum of the M-type giant. To detect the actual emission lines, removal of the underlying spectrum of the M-type giant from CH Cygni was carried out similar to that proposed in Paper II. The HD18191 (RZ Arietis, M6 III) spectrum was used as the standard.

The resultant line profiles of $\mathrm{H} \alpha$ are shown in the upper portion of Fig. 1. The resulting profile of the $\mathrm{H} \alpha$ emission lines might have at least four emission components and no absorption components as in October 2005 and June 2006. The intensities of the $\mathrm{H} \alpha$ emission lines on October 13, 2005, and June 4, 2006 are evidently less intense by about two times than and similar to those in October 2004, respectively. The widths of the $\mathrm{H} \alpha$ emission lines on October 13, 2005, and June 4, 2006, are less than those in 2004. It implies that the clouds forming $\mathrm{H} \alpha$ in October 2005 evidently changed to a smaller area than those forming $\mathrm{H} \alpha$ in October 2004.

The resulting line profiles for $\mathrm{H} \beta 4861 \AA$, [O III] $5007 \AA$, [Ne III] $3869 \AA$, and [S II] $4069 \AA$ are obtained in the same manner as mentioned above and are provided in the upper panels in Figs. 2 - 5. They do not appear 


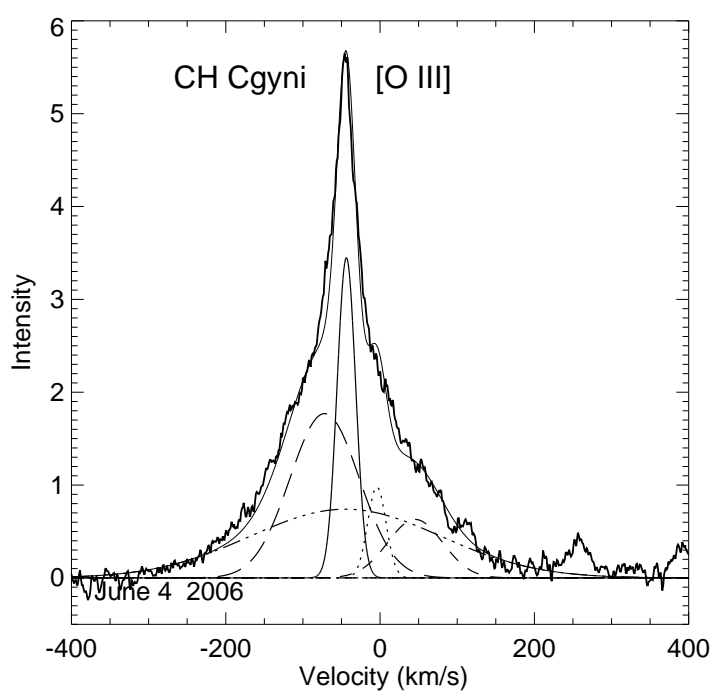

Fig. 7.- Gaussian deconvolution for the resultant [O III] $5007 \AA$ on June 4, 2006. The solid line denotes the observed line. The thin solid, the dashed, the dotted, the solid, and the dash dotted lines mean the result of fitting and each Gaussian component.

to be greatly affected even after the subtraction of the spectrum of the M-type giant. H8 and Ca II K lines are observed around $3889 \AA$ and $3933 \AA$, respectively.

The resultant [O III] $5007 \AA$ had broad emission components and its intensity was more enhanced on June 4, 2006 than that on October 13, 2005.

The intensity of the strongest emission component of [Ne III] $3869 \AA$ in June 2006 was decreased by half than that on October 13, 2005.

However, the intensity of the strongest emission component of [S II] $4069 \AA$ on October 13, 2005, was weaker by about $10 \%$ than that on June 4, 2006. The profiles of $[\mathrm{S} \mathrm{II}]$ appeared to be asymmetric. [S II] $4076 \AA$ was clearly observed in the resulting subtraction spectra.

Intensities of the emission lines of the resultant $\mathrm{Na} I \mathrm{D}_{1}$ and $\mathrm{D}_{2}$ profiles became gradually stronger after 2004.

\section{GAUSSIAN FITTING}

In section 3, the spectral line variations of $\mathrm{CH}$ Cygni were described in detail. All the emission lines involving blue continua in these epochs were stronger than those in 2004.

Because all the emission lines observed during these observation epochs had complicated profiles with several sub-peaks, each component of an emission line, when decomposed, might be arisen from a cloudlet in a gas cloud. The cloudlet might be expanding into the circumference space around the system with its radial

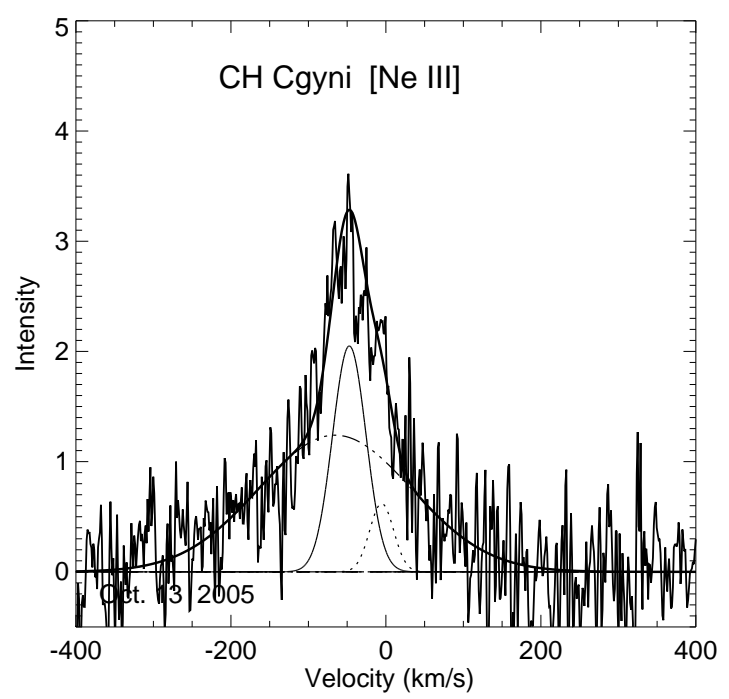

Fig. 8.- Gaussian deconvolution for the resultant [Ne III] $3869 \AA$ on October 13, 2005. The solid line denotes the observed line. The bold solid, the solid, the dotted, and the dash dotted lines mean the result of fitting and each Gaussian component.

velocity. Therefore, in this study, for the sake of simplicity, a likelihood model is assumed as a Gaussian function, and then the Gaussian deconvolution is carried out.

The emission lines are believed to be optically thin. Hence, if the velocity distribution of gas in the cloudlet is assumed as a Gaussian function, flux density emitted from the i-th cloudlet is represented by

$$
\begin{aligned}
F_{i} & =n_{e i} n_{i} \alpha \frac{h \nu}{4 \pi} V_{i} \phi_{i} \\
& =F_{0 i} \exp \left(-\left(\frac{\lambda-\lambda_{0 i}}{\sigma_{i}}\right)^{2}\right),
\end{aligned}
$$

where $\phi_{i}=\frac{1}{\sqrt{\pi} \sigma} \exp \left(-\left(\frac{\lambda-\lambda_{0 i}}{\sigma_{i}}\right)^{2}\right), \lambda_{0 i}=\lambda_{0}\left(1+\frac{v_{r i}}{c}\right)$, $F_{0 i}=n_{e i} n_{i} \alpha \frac{h \nu}{4 \pi} V_{i} \frac{1}{\sqrt{\pi} \sigma}, \lambda_{0}$ is the wavelength at the center of the function, $\mathrm{n}_{e}$ an electron density, $\mathrm{n}$ an ion density, $\alpha$ an recombination coefficient, $\mathrm{h}$ the Planck constant, $\nu$ a frequency of the line, $V_{i}$ a fractional part i of the emitting volume, and $\sigma$ a standard deviation, and $v_{r i}$ the radial velocity of the cloudlet i.

If there are $\mathrm{n}$ cloudlets in the cloud, total flux density is given by

$$
\begin{aligned}
F & =\Sigma F_{i} \\
& =\Sigma F_{0 i} \exp \left(-\left(\frac{\lambda-\lambda_{0 i}}{\sigma_{i}}\right)^{2}\right) .
\end{aligned}
$$

Here the sum of $i$ is the summation of $n$ cloudlets. 


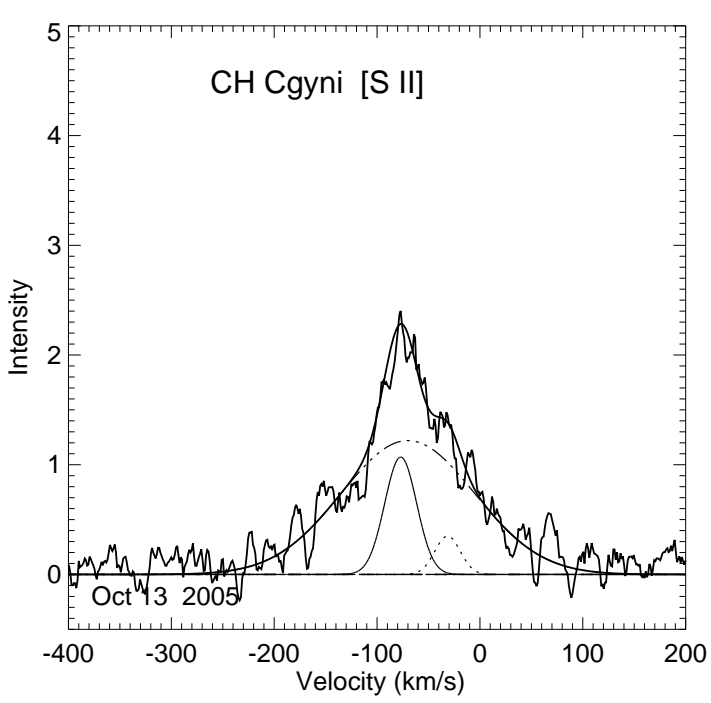

Fig. 9.- Gaussian deconvolution for the resultant [S II] $4069 \AA$ on October 13, 2005. The solid line denotes the observed line. The bold solid, the solid, the dotted, and the dash dotted lines mean the result of fitting and each Gaussian component.

It is uncertain that all the emission lines could be decomposed by Gaussian components. For example, the broad emission component of the [O III] nebular line might correspond with a rectangular line profile that the expanding shell structure produces. Therefore, it is believed that each of Gaussian components might be formed from either the expanding gas shell or a fine structure of the cloudlet. And, the doublepeaked profiles might be originated from the edge-on accretion disk (Robinson et al. 1994). The $\mathrm{H} \alpha$ and $\mathrm{H} \beta$ lines observed during these observation epochs had such profiles. They showed also complicated profiles with several sub-peaks, which might be originated from either a portion of the accretion disk or independently moving-out matter from the hot star.

Therefore, to investigate how these emission lines were formed, the observed emission lines were deconvoluted with several Gaussian functions. The Gaussian function is adopted from the equation of Ikeda and Tamura (2004). A program used for de-convolution of the observed lines into Gaussian functions was written by Dr. J.C. Chae.

Examples of the Gaussian deconvoluted line profiles of the resulting $\mathrm{H} \alpha$ and [O III] $5007 \AA$ spectra on June 4, 2006, and of the resulting [Ne III] $3869 \AA$ and [S II] $4069 \AA$ spectra on October 13, 2005 are presented in Figs.6-9 , respectively. Velocities in the abscissa of Figs. 6 - 9 are corrected to the sun.

The parameters of the Gaussian function are listed in Table 2. In Table $2, \lambda_{0}$ is the wavelength at the center of the deconvoluted Gaussian function; I is the peak intensity of the Gaussian function from a local continuum; $\mathrm{V}_{r}$ denotes the radial velocity; and $\mathrm{V}_{D}$ is the Doppler velocity of each Gaussian component.

During the present epochs, the Doppler velocities of the broad components, Ebr, of $\mathrm{H} \alpha$ on October 13, 2005, and on June 4, 2006 were smaller than those of the respective components in October 2004. In addition, the intensity of the strongest component, Em, of $\mathrm{H} \alpha$ was smaller by about 0.4 times on October 13, 2005, and was larger by about 1.1 times on June 4, 2006, than that of the corresponding line in October 2004.

The resultant synthetic line profile is well consistent with the simplified likelihood model of the Gaussian functions. It is a reason why the observed line profile, the most fiducial line profile, must be based on even in a comparison with any of many theoretical models. However, the broad emission wings of the observed $\mathrm{H} \alpha$ line might be the sum of several of such a rectangle profile. It means that the accretion disk might consist of several ring structures.

The Doppler velocities of the broad components, Ebr, of [O III] $5007 \AA$ and [Ne III] $3869 \AA$ on October 13, 2005 and on June 4, 2006 became larger than those of the respective components in October 2004. Intensities of the strongest component, Em, of [O III] $5007 \AA$ and [Ne III] $3869 \AA$ on October 13, 2005, and on June 4, 2006 became lower than those of the same of in October 2004. This implies that these elements were placed next to each other, and were kinematically affected by each other.

On October 13, 2005, and on June 4, 2006, the Doppler velocities of the broad components, Ebr, of $[\mathrm{N} \mathrm{II}]$, and $[\mathrm{S} \mathrm{II}]$ were about two hundred $\mathrm{km} \mathrm{s}^{-1}$.

\section{RADIAL VELOCITIES}

After the subtraction of the M-type spectrum, the line center could not be exactly decided even for the line peak of the strongest emission component. The radial velocities were measured around the center of the line peak at about $50 \%-90 \%$ of line intensities. Hence, there were small differences in the radial velocities between the observed lines and the Gaussian emission components for the referred lines, which range from $1-3 \mathrm{~km} \mathrm{~s}$. Discrepancies in the widths of the small sharp humps in both observed and resulting line profiles might be more than about $0.07 \AA$.

The radial velocities of the emission lines of $\mathrm{H} \mathrm{I}$, [O I], [O III], [Ne III], [Ca II], [N II], and [S II] were measured. Tables 3, 4 and 5 present the radial velocities measured for emission lines, forbidden lines, and absorption components, respectively. All the velocities are corrected to the Sun. When several lines were measured, the probable errors are quoted. 
TABle 2.

PARAMETERS OF THE GAUSSIAN COMPONENTS FOR THE ELEMENT OF CH CYGN

\begin{tabular}{|c|c|c|c|c|c|c|c|c|}
\hline Date & Element & $\begin{array}{c}\text { wavelength } \\
(\AA)\end{array}$ & Component & $\begin{array}{l}\lambda_{0} \\
(\AA)\end{array}$ & $\mathrm{I}$ & $\begin{array}{c}\text { FWHM } \\
(\AA)\end{array}$ & $\begin{array}{c}V_{r} \\
\left(\mathrm{~km} \mathrm{~s}^{-1}\right)\end{array}$ & $\begin{array}{c}V_{D} \\
\left(\mathrm{~km} \mathrm{~s}^{-1}\right)\end{array}$ \\
\hline \multirow[t]{3}{*}{ Oct. 13,2005} & \multirow[t]{3}{*}{$\mathrm{H} \alpha$} & \multirow[t]{3}{*}{6562.817} & Ebr & 6561.69 & 0.34 & 3.42 & -51.8 & 188.5 \\
\hline & & & Em & 6561.69 & 0.72 & 0.58 & -51.8 & 31.9 \\
\hline & & & E1 & 6562.12 & 0.60 & 0.57 & -32.0 & 31.4 \\
\hline \multirow{4}{*}{ June 04, 2006} & & & E1 & 6560.67 & 0.31 & 1.00 & -98.5 & 55.0 \\
\hline & & & Ebr & 6561.64 & 0.61 & 4.15 & -53.7 & 228.4 \\
\hline & & & Em & 6561.78 & 1.64 & 0.59 & -47.7 & 22.5 \\
\hline & & & $\mathrm{E} 2$ & 6562.74 & 0.16 & 1.14 & -3.8 & 63.0 \\
\hline \multirow[t]{3}{*}{ Oct. 13,2005} & \multirow[t]{3}{*}{$\mathrm{H} \beta$} & \multirow[t]{3}{*}{4961.332} & Ebr & 4860.48 & 1.20 & 3.42 & -52.6 & 254.5 \\
\hline & & & Em & 4860.48 & 5.10 & 0.80 & -52.6 & 59.4 \\
\hline & & & $\mathrm{E} 1$ & 4860.82 & 0.21 & 0.35 & -31.4 & 26.2 \\
\hline \multirow[t]{4}{*}{ June 04,2006} & & & E1 & 4859.75 & 0.78 & 0.97 & -97.6 & 71.8 \\
\hline & & & Ebr & 4860.48 & 0.65 & 3.85 & -52.3 & 286.1 \\
\hline & & & Em & 4860.54 & 3.02 & 0.60 & -48.8 & 44.6 \\
\hline & & & E2 & 4861.28 & 1.11 & 0.99 & -3.5 & 73.6 \\
\hline \multirow[t]{4}{*}{ Oct. 13,2005} & \multirow[t]{4}{*}{ [O III $]$} & \multirow[t]{4}{*}{5006.84} & E1 & 5005.30 & 0.97 & 0.70 & -92.5 & 50.6 \\
\hline & & & Ebr & 5005.87 & 1.04 & 4.47 & -58.4 & 322.6 \\
\hline & & & Em & 5005.94 & 1.95 & 0.62 & -53.6 & 44.7 \\
\hline & & & $\mathrm{E} 2$ & 5006.52 & 1.52 & 0.69 & -19.5 & 50.1 \\
\hline \multirow[t]{5}{*}{ June 04,2006} & & & E1 & 5005.52 & 1.77 & 1.76 & -78.8 & 127.4 \\
\hline & & & Ebr & 5005.98 & 0.74 & 4.86 & -51.7 & 350.6 \\
\hline & & & Em & 5006.00 & 3.45 & 0.47 & -50.6 & 33.9 \\
\hline & & & $\mathrm{E} 2$ & 5006.65 & 0.98 & 0.47 & -11.1 & 33.9 \\
\hline & & & E3 & 5007.48 & 0.63 & 1.40 & 38.5 & 100.8 \\
\hline \multirow[t]{3}{*}{ Oct. 13,2005} & [Ne III] & 3868.74 & Ebr & 3867.97 & 1.24 & 3.00 & -65.6 & 280.6 \\
\hline & & & Em & 3868.13 & 2.05 & 0.66 & -47.2 & 61.3 \\
\hline & & & E1 & 3868.67 & 0.62 & 0.47 & -5.4 & 43.9 \\
\hline \multirow{3}{*}{ June 04, 2006} & & & Ebr & 3867.94 & 0.54 & 2.02 & -62.4 & 188.5 \\
\hline & & & $\mathrm{Em}$ & 3868.03 & 1.05 & 0.47 & -55.1 & 43.9 \\
\hline & & & E1 & 3868.44 & 0.28 & 0.43 & -23.0 & 39.8 \\
\hline Oct. 13,2005 & $\mathrm{Na} I \mathrm{D}$ & 5889.953 & Ab1 & 5887.57 & 0.66 & 0.45 & -121.2 & 27.8 \\
\hline & & & $\mathrm{Ab} 2$ & 5887.97 & 0.62 & 0.44 & -101.0 & 26.9 \\
\hline & & & Ab3 & 5888.46 & 0.54 & 0.16 & -76.0 & 10.0 \\
\hline & & & E1 & 5888.84 & 0.35 & 0.33 & -56.7 & 20.3 \\
\hline & & & $\mathrm{E} 2$ & 5889.15 & 0.22 & 0.30 & -40.5 & 18.3 \\
\hline & & 5895.923 & Ab1 & 5893.53 & 0.44 & 0.24 & -122.0 & 15.0 \\
\hline & & & $\mathrm{Ab} 2$ & 5893.83 & 0.94 & 0.37 & -106.7 & 22.9 \\
\hline & & & Ab3 & 5894.41 & 0.67 & 0.18 & -76.8 & 11.1 \\
\hline & & & E1 & 5894.78 & 0.27 & 0.29 & -58.3 & 17.5 \\
\hline & & & E2 & 5895.16 & 0.22 & 0.33 & -39.0 & 20.3 \\
\hline June 04,2006 & $\mathrm{Na} I \mathrm{D}$ & 5889.953 & Ab1 & 5887.59 & 0.54 & 0.29 & -120.4 & 18.0 \\
\hline & & & Ab2 & 5887.91 & 0.88 & 0.34 & -104.1 & 21.0 \\
\hline & & & Ab3 & 5888.30 & 0.51 & 0.14 & -83.9 & 8.4 \\
\hline & & & E1 & 5888.66 & 0.19 & 0.39 & -65.7 & 24.0 \\
\hline & & & $\mathrm{E} 2$ & 5889.02 & 0.11 & 0.25 & -47.5 & 15.2 \\
\hline & & 5895.923 & Ab1 & 5893.72 & 0.74 & 0.34 & -111.9 & 20.9 \\
\hline & & & Ab2 & 5893.95 & 0.44 & 0.39 & -100.3 & 23.9 \\
\hline & & & Ab3 & 5894.31 & 0.61 & 0.14 & -82.2 & 8.4 \\
\hline & & & E1 & 5894.63 & 0.17 & 0.39 & -65.9 & 23.9 \\
\hline & & & $\mathrm{E} 2$ & 5895.00 & 0.11 & 0.25 & -46.7 & 15.2 \\
\hline Oct. 13,2005 & $\mathrm{Ca}$ II & 3933.664 & Em & 3932.09 & 1.01 & 0.15 & -120.4 & 14.1 \\
\hline & & & Ebr & 3933.02 & 2.02 & 0.38 & -49.2 & 34.7 \\
\hline June 04,2005 & & & Em & 3932.91 & 1.23 & 0.39 & -57.3 & 36.2 \\
\hline Oct. 13,2005 & {$[\mathrm{~S}$ II $]$} & 4068.62 & Ebr & 4067.79 & 1.22 & 2.07 & -60.8 & 183.9 \\
\hline & & & $\mathrm{Em}$ & 4067.70 & 1.07 & 0.50 & -67.9 & 44.1 \\
\hline & & & E1 & 4068.32 & 0.35 & 0.40 & -22.3 & 35.1 \\
\hline June 04,2006 & & & Ebr & 4067.88 & 0.67 & 2.24 & -54.6 & 199.0 \\
\hline & & & $\mathrm{Em}$ & 4067.71 & 0.98 & 0.42 & -67.1 & 37.0 \\
\hline & & & $\mathrm{E} 1$ & 4068.24 & 0.47 & 0.38 & -28.2 & 33.9 \\
\hline June 04,2006 & {$[\mathrm{Ne}$ III $]$} & 6583.6 & Em & 6582.22 & 0.08 & 1.12 & -62.7 & 61.4 \\
\hline & & & Ebr & 6582.17 & 0.11 & 3.79 & -65.3 & 208.2 \\
\hline
\end{tabular}

Ab1, Ab2, Ab3: Absorption components.

Ebr: The broadest emission component.

Ebr: The broadest emission component. 
TABLE 3.

RADIAL VELOCITIES $\left(\mathrm{km} \mathrm{s}^{-1}\right)$ MEASURED FOR OBSERVED EMISSION LINES

\begin{tabular}{ccccccc}
\hline \hline \multicolumn{1}{c}{ Date } & $\mathrm{H} \alpha$ & $\mathrm{H} \beta$ & $\mathrm{H} \gamma$ & Ca II H \& K & \multicolumn{2}{c}{ Fe II (Number of lines) } \\
\hline Oct. 13, 2005 & -51.3 & -52.7 & -52.6 & $-41.7 \pm 1.6$ & $-67.3 \pm 1.9$ & $(3)$ \\
June 4, 2006 & $-107.0,-44.1$ & -51.6 & -55.7 & $-47.7 \pm 1.6$ & -65.1 & $(1)$ \\
\hline
\end{tabular}

TABLE 4.

RADiAL VELOCITIES $\left(\mathrm{km} \mathrm{s}^{-1}\right)$ MEASURED FOR FORBIDDEN LINES

\begin{tabular}{cccccccc}
\hline \hline Date & {$[\mathrm{O} \mathrm{I}]$} & {$[\mathrm{O}$ III $]$} & {$[\mathrm{S} \mathrm{II}]$} & {$[\mathrm{N} \mathrm{II}]$} & {$[\mathrm{Ne}$ III $]$} & {$[\mathrm{Fe} \mathrm{II}]$ (Number of lines) } \\
& $6300 \AA$ & $4959,5007 \AA$ & $4069 \AA$ & $6582 \AA$ & $3869 \AA$ & & \\
\hline Oct. 13, 2005 & -65.2 & $-52.3 \pm 0.4$ & -69.0 & & $-49.7 \pm 1.9$ & $-65.5 \pm 2.1$ & $(4)$ \\
June 4, 2006 & -66.6 & $-52.2 \pm 0.6$ & -68.3 & -56.7 & -56.7 & $-71.6 \pm 2.1$ & $(4)$ \\
\hline
\end{tabular}

\section{DISCUSSION AND CONCLUSION}

The model involving an accretion disk and jets is introduced to interpret the characteristics of each of the lines identified in October 2005 and June 2006.

After 2004, the intensities and the line profiles of $\mathrm{H} \alpha$ continuously changed. For example, for $\mathrm{H} \alpha$, the $\mathrm{V} / \mathrm{R}$ was $<1$ in April 2004, $<<1$ in October 2004, $<1$ in October 2005, and $\sim 0.1-0.2$ in June 2006. Here, $\mathrm{V}$ and $\mathrm{R}$ represent the intensities of the blueward and redward peaks of $\mathrm{H} \alpha$. In October 2005, the $\mathrm{H} \alpha$ and $\mathrm{H} \beta$ lines had single-peaked emission lines, implying the existence of out-flowing radio jets. And the $\mathrm{H} \alpha$ line was weaker than the $\mathrm{H} \beta$ line. In June 2006, their intensities were opposed to each other. Conspicuous variations in the H I line profiles of CH Cygni from October 2004 to June 2006 might result from the collapse of the accretion disk and its subsequent formation. On the contrary, intensities of $\mathrm{H} \beta$ lines in 1983, one year before the 1984 eclipse on $\mathrm{CH}$ Cygni, were weaker than those in 1984, the beginning epoch of the eclipse (Hack et al., 1988).

In general, in October 2005 and June 2006, the shapes of the lines in the resultant $\mathrm{H} \alpha$ were widely different.

As shown in Fig. 10, from April 2004 to October 2004, the peak intensity, I, of the strongest emission components, Em, of the resulting $\mathrm{H} \alpha$ increased, whereas from October 2004 to October 2005, the I of Em of the resulting $\mathrm{H} \alpha$ decreased, and after those two epochs, the I of Em of the resulting $\mathrm{H} \alpha$ increased.

As shown in Fig. 11, the Doppler velocities, $V_{D}$, of the broad emission components, Ebr, of the resulting $\mathrm{H} \alpha$ steeply increased from April 2004 to October 2004, whereas from October 2004 to October 2005, these Doppler velocities, $\mathrm{V}_{D}$, slowly decreased, and subsequently remained nearly unchanged. In October 2005, $500 \mathrm{~km} \mathrm{~s}^{-1}$ of the Doppler velocity of $\mathrm{H}$ I was above the shock speed.
From these observations, the story regarding the evolution of CH Cygni is hypothesized: in April 2004, an accretion disk around the hot star was heading in the direction of its destruction; in October 2004 and October 2005, the jets were out-flowing; in June 2006 , the accretion disk adopted a destruction mode.

Results of decomposing the $\mathrm{H} \alpha$ line in 2006 are sketched. The blue component, E1, at $6560.7 \AA$ and the strongest one, Em, at $6561.8 \AA$ are originated from the accretion disk. The another one, E2, might be a fine structure in the disk.

During the past eruption phases, the blue continuum was believed to be produced due to the accretion disk (Yoo and Yamashita, 1984). However, Contini et al. (2008) suggested the UV lines were formed in expanding shocked nebulae.

According to Shakura and Sunyaev (1973), the coefficient of kinematic viscosity $\nu$ is represented as $\alpha \mathrm{C}_{s} H$. Here, $\alpha$ is a viscosity parameter, $\mathrm{C}_{s}$ the sound velocity, and $H$ a scale height of the disk in the z-direction. And, a viscous time scale is $\frac{R^{2}}{\nu} \sim \frac{R}{V_{r}}$. $R$ is the radius of the disk. If using radial velocities of the emission components of the $\mathrm{H} \alpha$ line and a standard $\alpha$-disk model, $\left|\mathrm{V}_{r}\right| \sim \alpha \mathrm{C}_{s} \frac{H}{R}>1$. Since $0<\alpha<1, H>R$. This indicates the accretion disk was newly born. In case an accretion disk was newly formed, it is expected to have been born between October $2005(\phi=0.52)$ and June $2006(\phi=0.57)$. Assume that only emission components $\mathrm{E} 1$ and $\mathrm{Em}$ of the $\mathrm{H} \alpha$ line consist of a new resulting profile of a $\mathrm{H} \alpha$ line. If so was it, FWHM of the resulting profile of a $\mathrm{H} \alpha$ line might be correspondent to the full separation of the double peaks of the $\mathrm{H} \alpha$. Since $\mathrm{FWHM}=\mathrm{V}_{o}$ sin $\mathrm{i}$, the outer radius of the accretion disk, $\mathrm{r}_{o}$, is approximately $7 \times 10^{8} \mathrm{~km}$, which is within the Lagrangian point. Here, the inclination of the accretion disk, $\mathrm{i}$, is adopted by $80^{\circ}$ (Robinson et al. 1994; Skopal 1995). From the radial velocities of $\mathrm{E} 1$ and $\mathrm{Em}$ of the $\mathrm{H} \alpha$, assume that an orbital velocity of an inner region of the accretion disk was about 25 
TABLE 5.

RADIAL VELOCITIES $\left(\mathrm{km} \mathrm{s}^{-1}\right)$ MEASURED FOR ABSORPTION LINES

\begin{tabular}{ccccc}
\hline \hline Date & Ca II & Na I & M-type (Number of lines) \\
& $\mathrm{H} \& \mathrm{~K}$ & $\mathrm{D}_{1}$ and $\mathrm{D}_{2}$ & & \\
\hline Oct. 13, 2005 & $-110.9 \pm 0.1$ & $-108.8 \pm 2.9-75.7 \pm 0.4$ & $-59.6 \pm 0.9$ & $(11)$ \\
June 4, 2006 & & $-110.9 \pm 1.0-79.3 \pm 1.0$ & $-50.4 \pm 0.6$ & $(10)$ \\
\hline
\end{tabular}

TABLE 6.

Normalized intensities AND Doppler velocities of THE RESUltant H $\alpha$, [O III] AND [Ne III] IN APRIL 9,2004 AND OCTOBER 2, 2004

\begin{tabular}{ccccc}
\hline \hline & \multicolumn{2}{c}{ I } & \multicolumn{2}{c}{$\mathrm{V}_{D}\left(\mathrm{~km} \mathrm{sec}^{-1}\right)$} \\
& Apr. 9, 2004 & Oct. 2, 2004 & Apr. 9, 2004 & Oct. 2, 2004 \\
\hline H $\alpha$ & 0.2 & 1.1 & 262 & 502 \\
{$[\mathrm{O}$ III] $5007 \AA$} & 2.9 & 8.4 & 100 & 148 \\
{$[\mathrm{Ne}$ III] } & - & 1.2 & - & 56 \\
\hline
\end{tabular}

All data are adopted from Figs 8 and 10 of Yoo (2007),

and Tables 1, 2 and 3 of Yoo (2007).

- means out of range of the spectrum in April, 2004

$\mathrm{km} \mathrm{s}^{-1}$. From this, its inner radius could be estimated as $10 \%-20 \%$ of its outer radius.

The resulting $\mathrm{Ca}$ II $\mathrm{H}$ and $\mathrm{K}$ lines in October, of both years 2004 and 2005, showed the P Cygni profiles, whereas that in June 2006 did not present the characteristic profile. The emission components of the resultant $\mathrm{Ca}$ II $\mathrm{H}$ and $\mathrm{K}$ lines were observed when $\mathrm{H} \alpha$ had a single-peaked profile. Hence, the emission components of the resultant Ca II lines might have originated in the low density region around the out-flowing radio jets.

The radial velocity of the [O I] $6300 \AA$ was used to arrive at the systemic velocity (Wallerstein, 1983). Its radial velocities did not appear to have changed in 2004 . Nevertheless, on October 13, 2005, and on June 4, 2006 , changes in the [O I] line profiles were observed. This implies that even the circumference cloud that formed the [O I] lines was symmetric in 2004, being affected by the radio jets that arose in both October 2004 and October 2005.

Using Tables 2 and 6 in this study, In Table 6,line intensities and expansion velocities of Gaussian components of the $\mathrm{H} \alpha$, [Ne III] and [O III] lines with respect to the observation dates are plotted in Figs. 10 and 11. I presents the peak intensity of the Gaussian component in unites of a local continuum, and $\mathrm{V}_{D}$ denotes the Doppler velocity of the Gaussian component.

As shown in Figs. 10 and 11, in the present epochs, the resulting [O III] lines might have more than four emission Gaussian components. [O III] lines were expected to be formed in the several circumference cloudlets around the hot star of CH Cygni. Just after 2004, the Doppler velocity of the broad component of the resulting [O III] line appeared to have rapidly increased, and the peak intensity of its strongest emission component also gradually increased. It indicates that the cloudlets emitting the [O III] lines might be moved out by the out-flowing radio jets. For example, the broadest emission component, Ebr, and the sharper main emission component, Em, of [O III] 5007 $\AA$ in June 2006 were formed by the jets in October 2005 and by jets in October 2004, respectively. The sharper main emission component, Em, of [O III] $5007 \AA$ in June 2006 might be due to the collimated jet streams in October 2004. In this context, AG Pegasi showed differences in the radial velocities of many of the permitted lines due to the occurrence of the shock waves (Yoo, 2008).

The resultant [Ne III] lines appeared to have at least three emission components. As shown in Fig. 10 and 11, from April 2004 to October 2005, the Doppler velocities, $\mathrm{V}_{D}$, of the broad emission components of the resulting $[\mathrm{Ne}$ III] and the resulting [O III] were gradually increased, whereas from October 2005 to June 2006 , that of [Ne III] varied with a gradient opposite to that of [O III]. This implies that the [Ne III] clouds took the important role of transporting their momenta to the [O III] clouds. The decrease in the velocities of the [Ne III] clouds results in a little increase in their optical thickness during the period from October 2005 to June 2006. The [Ne III] clouds helped the strongest emission component, Em, of the [O III] $5007 \AA$ to become even sharper. From these discussions, the clouds forming the [Ne III] line might be concluded to be located inside the clouds emitting the [O III] line.

The $[\mathrm{S} \mathrm{II}]$ line profiles were asymmetric and had 


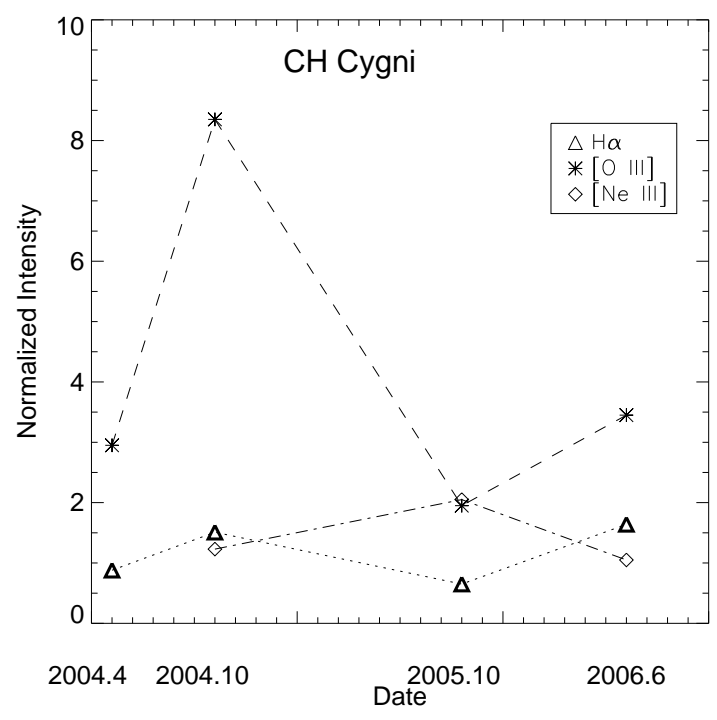

Fig. 10.- Normalrized intensities in units of local continuum of resulting Gaussian functions of $\mathrm{H} \alpha$, [O III] and [Ne III] with the dates. Weak intensity components are excluded.

more than three emission components. This implies that the $[\mathrm{S} \mathrm{II}]$ clouds originated in the circumference material at the outskirts of an ellipsoidal envelope that was formed as a result of the out-flowing radio jets.

The emission components of the resulting $\mathrm{Na}$ I D lines became enhanced from 2004 to the present epochs. Their radial velocities were in agreement with those of $\mathrm{H} \alpha$. Hence, $\mathrm{Na}$ I D and $\mathrm{Ca}$ II H and K emission components might be also formed because of the out-flowing radio jets. The absorption components of $\mathrm{Na} \mathrm{I} \mathrm{D}$, whose radial velocities were about $-77 \mathrm{~km} \mathrm{sec}^{-1}$, might have their origin in the M-type star.

It is well known that a leading cause for the formation of $\mathrm{Na} I \mathrm{D}$ lines is spin-orbital interactions of the electrons of $\mathrm{Na} \mathrm{I}$ in $1.84 \times 10^{5} \mathrm{G}$ of the strong magnetic field around the hot star of $\mathrm{CH}$ Cygni. Assuming the surface magnetic field of a hot star was about $10^{7} \mathrm{G}$, and the hot star had a dipole magnetic field, the magnetic field falls off as $\mathrm{B} \sim \mathrm{B}_{*}\left(\frac{R_{*}}{r}\right)^{3}$, where $\mathrm{R}_{*}$, the radius of the hot star, is the $0.01 R_{\odot}$, and $B_{*}$ its surface magnetic field. Hence, the region forming the Na I D lines is placed approximately at $2.64 \times 10^{4} \mathrm{~km}$, which is unexpectedly close to the hot star. Assuming this distance to be an Alfven radius and the spherical symmetric radial inflow of matter toward the hot star, the accretion rate needed forming the $\mathrm{Na}$ I D lines could be adopted as approximately $10^{-6} \mathrm{M}_{\odot} /$ year, which are larger by about 10 times than the estimated accretion rate of the upper limit.

A brief scenario in this study is as follows. According to the AAVSO, CH Cygni was fainter on October 13, 2005 than on June 4, 2006. The pulsating M III star in the the companion star of $\mathrm{CH}$ Cygni had a larger ra-

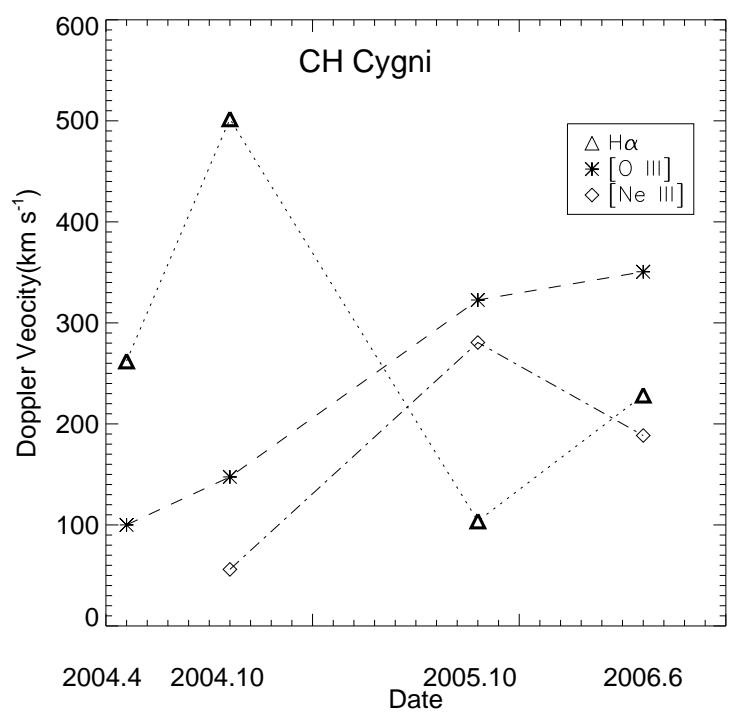

Fig. 11. - Doppler velocities of resulting broad Gaussian functions of $\mathrm{H} \alpha,[\mathrm{O} \mathrm{III}]$ and $[\mathrm{Ne} \mathrm{III}]$ with the dates.

dius on October 13, 2005 than on June 4, 2006. Around October 13, 2005, the stellar matter of the M III star was transferred to the hot star, forming an accretion disk around the hot star on around June 4, 2006. However, no observational data is available as to when the accretion disk that appeared on October 13, 2005, collapsed. The $\mathrm{H} \alpha$ lines of CH Cygni in April 2004 had double emission peaks, characteristic of an accretion disk. On October 13, 2005, and on June 4, 2006, many elements within the circumference of $\mathrm{CH}$ Cygni might have been on head-on collision courses.

\section{ACKNOWLEDGEMENTS}

The authors would be grateful to Professor Yamashita for his helpful comments of improving this work, and also thank the referee for some comments that improved this paper. We acknowledge with thanks the variable star observations from the AAVSO International Database provided the light curve of $\mathrm{CH}$ Cygni. The authors appreciate all the help and kindness of BOAO staff and observation operators during their stay for observations.

\section{REFERENCES}

Contini, M., Angeloni, R., \& Rafanelli, P., 2008, The symbiotic star $\mathrm{CH}$ Cygni. I. An analysis of the shocked nebulae at different epochs, arXiv 0807, 1480

Eyres, S. P. S., Bode, M. F., Skopal, A., Crocker, M. M., Davis, R. J., Taylor, A. R., Teodorani, M., Errico, L., Vittone, A. A. \& Elkin, V. G., 2002, The symbiotic star CH Cygni-II. The ejecta from 19982000 active phase, MNRAS, 335, 526 
Faraggiana, R. \& Hack, M., 1969, Spectroscopic evidence for mass loss from CH Cgni, AP\&SS, 3, 205

Hack, M., Engin, S., Rusconi, L., Sedmak, G., Yilmaz, N., \& Boehm, C., 1988, Line spectrum variations during the CH Cygni outburst of 1977-1986, A\&AS, 72,391

Hack, M., Rusconi, L., Sedmak, G., Aydin, C., Engin, S., \& Yilmaz, N., 1986, CH Cygni-The outburst of 1977-85, A\&A, 159, 117

Hinkle, K. H., Fekel, F. C., Johnson, D. S., \& Scharlach, W. W. G., 1993, The triple symbiotic system CH Cygni, AJ, 105, 1074

Ikeda, Y. \& Tamura, S., 2004, Spectroscopic Diagnostics of Symbiotic Stars II. Statistical Analyses of Highly Resolved Emission Line Profiles, PASJ, 56, 353

Mikolajewski, M., Mikolajewska, J., \& Khudiakova, T. N., 1990, The long-period symbiotic binary $\mathrm{CH}$ Cygni, A\&A, 235, 219

Robinson, K., Bode, M. F., Skopal, A., Ivison, R. J., \& Meaburn, J., 1994, On the nature of the emissionline profiles of symbiotic stars-I. Accretion discs, MNRAS, 269, 1

Shakura, N. I. \& Sunyaev, R. A., 1973, Black holes in binary systems. Observational appearance, A\&A, 24, 337

Skopal, A., Bode, M. F., Crocker, M. M., Drechsel, H., Eyres, S. P. S., \& Komzik, R., 2002, The symbiotic star CH Cygni-IV. Basic kinematics of the circumstellar matter during active phases, MNRAS, 335, 1109

Skopal, A., 1995, Phtometric observations of eclipes in the symbiotic triple system CH Cygni, IBVS, 4157, 1

Wallerstein, G., 1983, CH Cygni 1981-82 - Now a shell star, PASP, 95, 135

Yamashita, Y., 1967, MK Spectral Types of Bright MType Stars, PDAO, 13, 47

Yamashita, Y. \& Maehara, H., 1979, A Binary Model for CH Cygni, PASJ, 31, 307

Yoo, K. H. \& Yamashita Y., 1984, Radial velocity components and their variations in the circumstellar spectrum of CH Cygni, PASJ, 36, 567

Yoo, K. H., 2006, The high resolution spectra of symbiotic star CH Cygni in 2004, NewA, 11, 359, Paper I

Yoo, K. H., 2007, CH Cygni in 2004 II: Nebular spectrum features, NewA, 12, 569, Paper II

Yoo, K. H., 2008, High resolution spectra of the symbiotic star AG Pegasi in 2004-2006, NewA, 13, 646 